\title{
Analytical Investigation of Laminar Viscoelastic Fluid Flow over a Wedge in the Presence of Buoyancy Force Effects
}

\author{
B. Rostami, ${ }^{1}$ M. M. Rashidi, ${ }^{1,2}$ P. Rostami, ${ }^{3}$ E. Momoniat, ${ }^{4}$ and N. Freidoonimehr ${ }^{5}$ \\ ${ }^{1}$ Mechanical Engineering Department, Engineering Faculty, Bu-Ali Sina University, Hamedan, Iran \\ ${ }^{2}$ University of Michigan-Shanghai Jiao Tong University Joint Institute, Shanghai Jiao Tong University, Shanghai, China \\ ${ }^{3}$ Department of Mechanical Engineering, Isfahan University of Technology, Isfahan 84156 83111, Iran \\ ${ }^{4}$ Centre for Differential Equations, Continuum Mechanics and Applications, School of Computational and Applied Mathematics, \\ University of the Witwatersrand (Wits), Private Bag 3, Johannesburg 2050, South Africa \\ ${ }^{5}$ Young Researchers \& Elite Club, Islamic Azad University, Hamedan Branch, Hamedan, Iran \\ Correspondence should be addressed to M. M. Rashidi; mm_rashidi@yahoo.com
}

Received 5 March 2014; Revised 18 April 2014; Accepted 21 April 2014; Published 21 May 2014

Academic Editor: Rehana Naz

Copyright (C) 2014 B. Rostami et al. This is an open access article distributed under the Creative Commons Attribution License, which permits unrestricted use, distribution, and reproduction in any medium, provided the original work is properly cited.

\begin{abstract}
An analytical strong method, the homotopy analysis method (HAM), is employed to study the mixed convective heat transfer in an incompressible steady two-dimensional viscoelastic fluid flow over a wedge in the presence of buoyancy effects. The twodimensional boundary-layer governing partial differential equations (PDEs) are derived by the consideration of Boussinesq approximation. By the use of similarity transformation, we have obtained the ordinary differential nonlinear (ODE) forms of momentum and energy equations. The highly nonlinear forms of momentum and energy equations are solved analytically. The effects of different involved parameters such as viscoelastic parameter, Prandtl number, buoyancy parameter, and the wedge angle parameter, which is related to the exponent $m$ of the external velocity, on velocity and temperature distributions are plotted and discussed. An excellent agreement can be seen between the results and the previously published papers for $f^{\prime \prime}(0)$ and $\theta^{\prime}(0)$ in some of the tables and figures of the paper for velocity and temperature profiles for various values of viscoelastic parameter and Prandtl number. The effects of buoyancy parameter on the velocity and temperature distributions are completely illustrated in detail.
\end{abstract}

\section{Introduction}

Since the non-Newtonian fluid flow and heat transfer represents many important applications such as plastic films and artificial fibers, it is one of the most attractive fields in different aspects of engineering for the last few decades. One of the important studies is related to the convective heat transfer over a surface which can be observed vastly in engineering, agriculture, and petroleum industries [1,2]. Hiemenz [3] was the first one who started the study of stagnation flow problem and obtained the ODE form of equations for the forced convective problem by introducing a similarity transformation. Dash and Behera [4] investigated laminar free convective viscoelastic fluid flow and heat transfer over an isothermal cylinder. Nazar et al. [5] studied the micropolar fluid flow over a stretching sheet in stagnation flow. Viscoelastic MHD flow and heat transfer over a stretching sheet was investigated by Abel et al. [6] considering viscous and ohmic dissipations. Nadeem and Akbar [7-10] solved different types of fluid flows such as non-Newtonian, Williamson, and tangent hyperbolic fluids in an endoscope analytically, numerically, and exactly. Presenting a numerical algorithm, Ariel [11] studied viscoelastic (second grade) fluid flow near a stagnation point. Finite difference method using Thomas algorithm was employed by Mahapatra and Gupta [12] to survey viscoelastic (Walters' B liquid) fluid flow. An off-centered stagnation flow over a rotating disc was solved by Erfani et al. [13] by the modified differential transform method (MDTM). Vogel's model of viscosity on the peristaltic flow of Jeffrey fluid was considered by Akbar et al. [14] in analytical and numerical forms. Ishak et al. [15] presented the results of stagnationpoint flow in a permeable sheet numerically via an implicit finite difference scheme known as the Keller-Box method. Rashidi et al. [16] also analyzed the stagnation-point flow in 
a porous medium by DTM. Kasim et al. [17] considered heat generation in a free convective viscoelastic fluid flow over a horizontal circular cylinder at the lower stagnation point of the cylinder. Aman et al. [18] considered the slip condition in mixed convective boundary-layer flow numerically. The unsteady three-dimensional stagnation-point flow of a viscoelastic fluid was studied by Seshadri [19]. Turkyilmazoglu [20] presented exact solution for mixed convection over a stretching surface. Bhattacharyya and Layek [21] presented numerically the results of suction/blowing and thermal radiation effects over a porous shrinking sheet. Bachok et al. [22] and Layek et al. [23] employed Runge-Kutta-Fehlberg and fourth order classical Runge-Kutta methods in the study of stretching/shrinking sheets, respectively. Turkyilmazoglu [24-26] presented multiple solutions in viscoelastic MHD fluid flow and heat and mass transfer over stretching surfaces in slip condition.

Unlike the number of studies related to stretching sheets, the articles on the convective flow over a wedge are very limited. A nonisothermal wedge in the presence of a heat source/sink was the base of investigation solved by Chamkha et al. [27] in the presence of thermal radiation effects by the finite-difference method. Kandasamy et al. [28] considered the effects of chemical reaction, variable viscosity, and thermophoresis over a porous wedge. Hossain et al. [29] presented the numerical results for unsteady flow over a symmetric wedge with variable surface temperature.

Homotopy analysis method (HAM) is one of the most well-known methods to solve highly nonlinear problems. The first one who employed HAM, Liao, offered a general analytical method for nonlinear problems [30, 31]. Rashidi et al. [32] used this method for mixed convective boundary-layer flow of a micropolar fluid towards a heated shrinking sheet. The stagnation-point flow of a nanofluid over a stretching sheet was investigated by Mustafa et al. [33] via HAM. Dinarvand et al. [34] used HAM to solve the unsteady laminar flow near stagnation point of a rotating and translating sphere. Hayat et al. [35] considered MHD flow of an upper-convected (UCM) fluid over a stretching surface by means of HAM. Currently, HAM has been used vastly by many researchers in different practical aspects of engineering and nonlinear problems [3642].

In the present paper, we try to find the analytical solution for two-dimensional incompressible viscoelastic fluid flow over a wedge. Analytical solutions for the velocity and the temperature distributions are obtained using a powerful technique, namely, the HAM. The graphs are plotted and discussed for the variations of different involved parameters.

\section{Flow Analysis}

Consider a steady and laminar incompressible two-dimensional mixed convective heat transfer of a viscoelastic fluid flow over a wedge in the presence of buoyancy force effects. It is assumed that the external velocity is in the form of $u_{e}(x)=$ $a x^{m}$ where $a$ and $m$ are constants. The Cartesian coordinate system is supposed to help the solution in which the $x$-axis and the $y$-axis are along with and perpendicular to the wedge

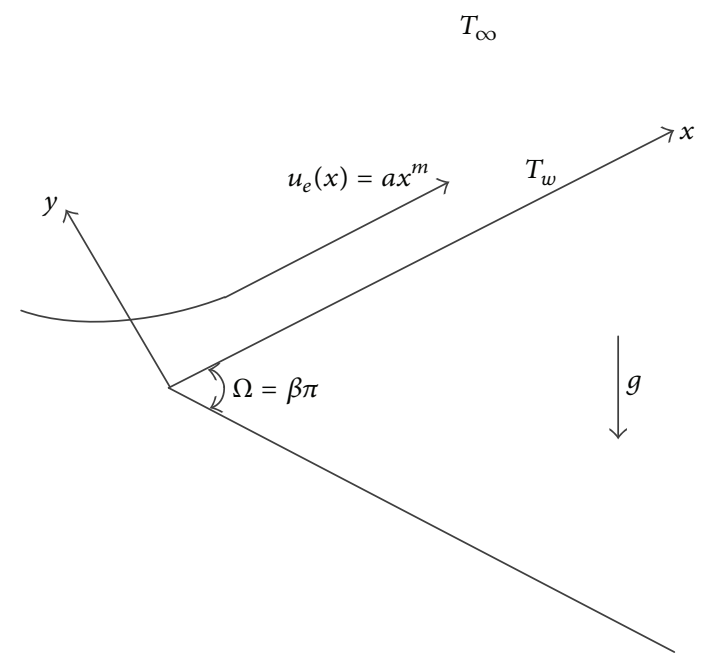

FIGURE 1: The schematic diagram of the wedge.

surface, respectively. The schematic diagram of the problem is plotted in Figure 1 in order to simplify the problem's realization. Considering the Boussinesq, the boundary-layer approximations, and the above assumptions, we can derive the boundary-layer equations in the following format:

$$
\begin{gathered}
\frac{\partial u}{\partial x}+\frac{\partial v}{\partial y}=0 \\
u \frac{\partial u}{\partial x}+v \frac{\partial u}{\partial y}=u_{e} \frac{d u_{e}}{d x}+v \frac{\partial^{2} u}{\partial y^{2}} \\
+k_{0}\left(u \frac{\partial^{3} u}{\partial x \partial y^{2}}+\frac{\partial u}{\partial x} \frac{\partial^{2} u}{\partial y^{2}}+\frac{\partial u}{\partial y} \frac{\partial^{2} v}{\partial y^{2}}+v \frac{\partial^{3} u}{\partial y^{3}}\right) \\
+g \beta_{T}\left(T-T_{\infty}\right) \operatorname{Sin} \frac{\Omega}{2} \\
u \frac{\partial T}{\partial x}+v \frac{\partial T}{\partial y}=\frac{k}{\rho c_{P}} \frac{\partial^{2} T}{\partial y^{2}}
\end{gathered}
$$

where $u$ and $v$ are velocity components in the directions of $x$ and $y$ (as shown in Figure 1). $v$ is the kinematic viscosity, $k_{0}$ is the viscoelasticity parameter, $g$ is the acceleration due to the gravity, $\beta_{T}$ is the coefficient of thermal expansion, $k$ is the thermal conductivity, $\rho$ is the fluid density, $c_{P}$ is the specific heat at constant pressure, and $T$ is the fluid temperature. $\Omega=$ $\pi \beta$ is the total angle of the wedge (for $\beta=0$ and $\beta=1$, the wedge takes the form of horizontal and vertical plate, resp.) and $\beta$ is the wedge angle parameter which is defined in the form of $\beta=2 m /(m+1)$.

The corresponding boundary conditions are as follows:

$$
\begin{gathered}
u=0, \quad v=0, \quad T=T_{w}(x), \quad \text { at } y=0, \\
u \longrightarrow u_{e}(x), \quad \frac{\partial u}{\partial y} \longrightarrow 0, \quad T \longrightarrow T_{\infty} \quad \text { as } y \longrightarrow \infty .
\end{gathered}
$$

It is assumed that $T_{w}(x)=T_{\infty}+b x^{m}$ where $b$ is constant value. After introducing the stream function $\psi$ and 
similarity variable $\eta$ and satisfying the continuity equation, we can derive the momentum and energy equations into the ordinary differential equations (ODEs) format as one can see in the following equations:

$$
\begin{gathered}
\eta=\sqrt{\frac{u_{e}(x)}{v x}} y, \quad \psi=\sqrt{v x u_{e}(x)} f(\eta), \quad \theta(\eta)=\frac{T-T_{\infty}}{T_{w}-T_{\infty}}, \\
m\left(f^{\prime 2}-1\right)-\frac{m+1}{2} f f^{\prime \prime}-f^{\prime \prime \prime} \\
-k_{1}\left\{(3 m-1) f^{\prime} f^{\prime \prime \prime}-\frac{(3 m-1)}{2} f^{\prime \prime 2}-\frac{(m+1)}{2} f f^{(4)}\right\} \\
-\lambda_{T} \operatorname{Sin}\left(\frac{m}{m+1} \pi\right) \theta=0, \\
\theta^{\prime \prime}+\operatorname{Pr}\left(\frac{m+1}{2} f \theta^{\prime}-m f^{\prime} \theta\right)=0,
\end{gathered}
$$

where superscript ' denotes the derivative with respect to $\eta$, $k_{1}=k_{0} a x^{m-1} / v$ is the viscoelastic parameter (when $m=1$, the viscoelastic parameter takes the form of $k_{1}=k_{0} a / v$ similar to the viscoelastic parameter obtained by Hayat et al. [44]), and $\lambda_{T}=g \beta_{T}\left(T_{w}-T_{\infty}\right) x /\left(a^{2} x^{(2 m-1)} x\right)=\mathrm{Gr}_{x} / \operatorname{Re}_{x}^{2}$ is the buoyancy parameter, where $\mathrm{Gr}_{x}=g \beta_{T}\left(T_{w}-T_{\infty}\right) x^{3} / v^{2}$ is the Grashof number, $\operatorname{Re}_{x}=u_{e} x / v=a x^{m+1} / v$ is the Reynolds number, and $\operatorname{Pr}=\mu c_{P} / k$ is the Prandtl number. The corresponding boundary conditions are as follows:

$$
\begin{gathered}
f(\eta)=0, \quad f^{\prime}(\eta)=0, \quad \theta(\eta)=1, \quad \text { at } \eta=0, \\
f^{\prime}(\eta)=1, \quad f^{\prime \prime}(\eta)=0, \quad \theta(\eta)=0, \quad \text { as } \eta \longrightarrow \infty .
\end{gathered}
$$

\section{HAM Solution}

In order to satisfy the boundary conditions, the initial approximations must be chosen in the appropriate form as follows:

$$
\begin{gathered}
f_{0}(\eta)=\eta+\left(e^{-\eta}-1\right), \\
\theta_{0}(\eta)=e^{-\eta} .
\end{gathered}
$$

The linear operators $\mathscr{L}_{f}(f), \mathscr{L}_{\theta}(\theta)$ are defined as

$$
\begin{gathered}
\mathscr{L}_{f}(f)=\frac{\partial^{4} f}{\partial \eta^{4}}+\frac{\partial^{3} f}{\partial \eta^{3}}, \\
\mathscr{L}_{\theta}(\theta)=\frac{\partial^{2} \theta}{\partial \eta^{2}}+\frac{\partial \theta}{\partial \eta},
\end{gathered}
$$

with the following properties:

$$
\begin{gathered}
\mathscr{L}_{f}\left(c_{1}+c_{2} \eta+c_{3} \eta^{2}+c_{4} e^{-\eta}\right)=0, \\
\mathscr{L}_{\theta}\left(c_{5}+c_{6} e^{-\eta}\right)=0,
\end{gathered}
$$

where $c_{1}-c_{6}$ are arbitrary constants. The nonlinear operators are

$$
\begin{aligned}
& \mathcal{N}_{f}[\widehat{f}(\eta ; q), \widehat{\theta}(\eta ; q)] \\
& =m\left(\left(\frac{\partial \widehat{f}(\eta ; q)}{\partial \eta}\right)^{2}-1\right) \\
& -\frac{m+1}{2} \widehat{f}(\eta ; q) \frac{\partial^{2} \hat{f}(\eta ; q)}{\partial \eta^{2}}-\frac{\partial^{3} \widehat{f}(\eta ; q)}{\partial \eta^{3}} \\
& -k_{1}\left\{(3 m-1) \frac{\partial \widehat{f}(\eta ; q)}{\partial \eta} \frac{\partial^{3} \widehat{f}(\eta ; q)}{\partial \eta^{3}}\right. \\
& -\frac{m+1}{2} \widehat{f}(\eta ; q) \frac{\partial^{4} \widehat{f}(\eta ; q)}{\partial \eta^{4}} \\
& \left.-\frac{3 m-1}{2}\left(\frac{\partial^{2} \widehat{f}(\eta ; q)}{\partial \eta^{2}}\right)^{2}\right\} \\
& -\lambda_{T} \operatorname{Sin}\left(\frac{m}{m+1} \pi\right) \hat{\theta}(\eta ; q), \\
& \mathcal{N}_{\theta}[\widehat{f}(\eta ; q), \widehat{\theta}(\eta ; q)] \\
& =\frac{\partial^{2} \widehat{\theta}(\eta ; q)}{\partial \eta^{2}} \\
& +\operatorname{Pr}\left(\frac{m+1}{2} \widehat{f}(\eta ; q) \frac{\partial \widehat{\theta}(\eta ; q)}{\partial \eta}-m \frac{\partial \widehat{f}(\eta ; q)}{\partial \eta} \widehat{\theta}(\eta ; q)\right)
\end{aligned}
$$

The auxiliary functions are introduced as

$$
\mathscr{H}_{f}(\eta)=\mathscr{H}_{\theta}(\eta)=e^{-\eta} \text {. }
$$

Now, the $i$ th order deformation equations (14) are solved by the symbolic software MATHEMATICA:

$$
\begin{aligned}
& \mathscr{L}_{f}\left[f_{i}(\eta)-\chi_{i} f_{i-1}(\eta)\right]=\hbar \mathscr{H}_{f}(\eta) R_{f, i}(\eta), \\
& \mathscr{L}_{\theta}\left[\theta_{i}(\eta)-\chi_{i} \theta_{i-1}(\eta)\right]=\hbar \mathscr{H}_{\theta}(\eta) R_{\theta, i}(\eta)
\end{aligned}
$$


where $\hbar$ is the auxiliary nonzero parameter:

$$
\begin{aligned}
& R_{f, i}(\eta) \\
& =\sum_{j=0}^{i-1}\left(m \frac{\partial f_{j}(\eta)}{\partial \eta} \frac{\partial f_{i-1-j}(\eta)}{\partial \eta}-\frac{m+1}{2} f_{j}(\eta) \frac{\partial^{2} f_{i-1-j}(\eta)}{\partial \eta^{2}}\right) \\
& -\frac{\partial^{3} f_{i-1}(\eta)}{\partial \eta^{3}}-m\left(1-\chi_{i}\right) \\
& -\sum_{j=0}^{i-1} k_{1}\left((3 m-1) \frac{\partial f_{j}(\eta)}{\partial \eta} \frac{\partial^{3} f_{i-1-j}(\eta)}{\partial \eta^{3}}\right. \\
& -\frac{m+1}{2} f_{j}(\eta) \frac{\partial^{4} f_{i-1-j}(\eta)}{\partial \eta^{4}} \\
& \left.-\left(\frac{3 m-1}{2}\right) \frac{\partial^{2} f_{j}(\eta)}{\partial \eta^{2}} \frac{\partial^{2} f_{i-1-j}(\eta)}{\partial \eta^{2}}\right) \\
& -\lambda_{T} \operatorname{Sin}\left(\frac{m}{m+1} \pi\right) \theta_{i-1}(\eta), \\
& R_{\theta, i}(\eta)=\frac{\partial^{2} \theta_{i-1}(\eta)}{\partial \eta^{2}} \\
& +\operatorname{Pr} \sum_{j=0}^{i-1}\left(\frac{m+1}{2} f_{j}(\eta) \frac{\partial \theta_{i-1-j}(\eta)}{\partial \eta}\right. \\
& \left.-m \theta_{j}(\eta) \frac{\partial f_{i-1-j}(\eta)}{\partial \eta}\right), \\
& \chi_{i}= \begin{cases}0, & i \leq 1, \\
1, & i>1 .\end{cases}
\end{aligned}
$$

For more information about the HAM solution, please see $[30,31]$.

In Figure $2 \hbar$-curves are plotted for an especial case, obtained via 20th order of HAM solution. Choosing a proper value of auxiliary parameter from the valid region in straight line is very important to control the convergence of the approximation series in the so-called $\hbar$-curve. To check the accuracy of the method, we have presented Tables 1-4 and Figures 3 and 4. An excellent agreement can be found between our results and the published papers.

In order to choose the optimal value of auxiliary parameter $\hbar$, we have presented the average residual error as (see [40, 45-47], for more details)

$$
\begin{aligned}
& \Delta_{f, m}=\frac{1}{K} \sum_{i=0}^{K}\left[\operatorname{Res}_{f}\left(\sum_{j=0}^{m} f_{j}(i \Delta x)\right)\right]^{2}, \\
& \Delta_{\theta, m}=\frac{1}{K} \sum_{i=0}^{K}\left[\operatorname{Res}_{\theta}\left(\sum_{j=0}^{m} \theta_{j}(i \Delta x)\right)\right]^{2},
\end{aligned}
$$

where $\Delta x=10 / K$ and $K=20$. For the given order of approximation $m$, the optimal value of $\hbar$ is given by the minimum

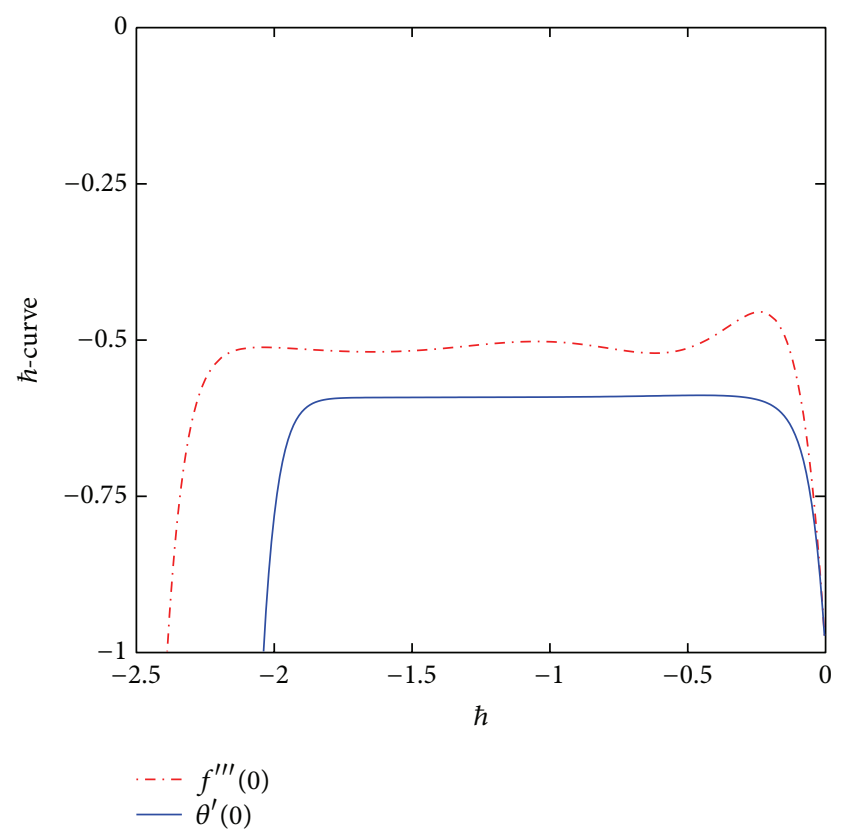

Figure 2: The $\hbar$-curves of $f \prime \prime(0)$ and $\theta^{\prime}(0)$ obtained by the 20th order approximation of the HAM solution when $k_{1}=1.0, \lambda_{T}=0.2$, $\operatorname{Pr}=1.0$, and $m=0.5$.

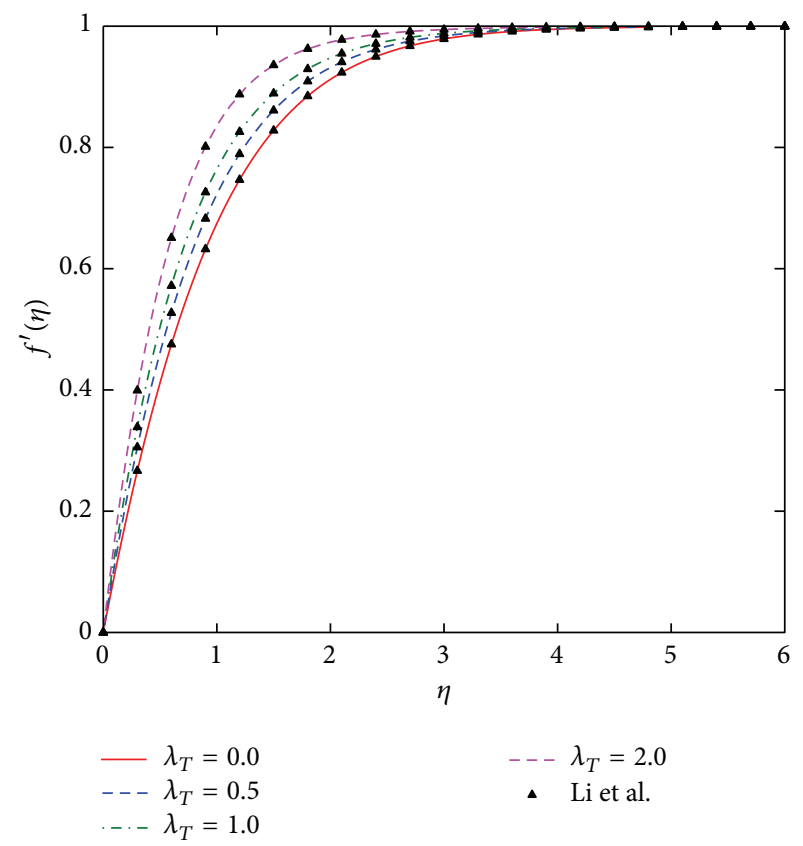

Figure 3: Verification of the results $f^{\prime}(\eta)$ with the previously published paper by Li et al. when $k_{1}=0.3, m=1.0$, and $\operatorname{Pr}=1.0$.

values of $\Delta_{f, m}$ and $\Delta_{\theta, m}$ corresponding to nonlinear algebraic equations:

$$
\frac{d \Delta_{f, m}}{d \hbar}=0, \quad \frac{d \Delta_{\theta, m}}{d \hbar}=0 .
$$


TABLE 1: Comparison of $f^{\prime \prime}(0)$ for various values of $k_{1}$ and Pr when $m=1.0$ and $\lambda_{T}=0.2$.

\begin{tabular}{|c|c|c|c|c|c|c|c|}
\hline \multirow{3}{*}{$k_{1}$} & \multirow{3}{*}{$\operatorname{Pr}$} & \multicolumn{6}{|c|}{$f^{\prime \prime}(0)$} \\
\hline & & \multicolumn{2}{|c|}{ Present results } & \multicolumn{2}{|c|}{ Li et al. [2] } & \multicolumn{2}{|c|}{ Hayat et al. [43] } \\
\hline & & Assisting flow & Opposing flow & Assisting flow & Opposing flow & Assisting flow & Opposing flow \\
\hline 0.0 & 0.2 & 1.35428 & 1.10711 & 1.35426 & 1.10711 & 1.3543 & 1.1072 \\
\hline 0.2 & & 1.15589 & 0.956065 & 1.15591 & 0.95607 & 1.1559 & 0.9558 \\
\hline 0.5 & & 0.982302 & 0.818537 & 0.98230 & 0.81854 & 0.9821 & 0.8184 \\
\hline 0.7 & & 0.904415 & 0.755544 & 0.90441 & 0.75554 & 0.9044 & 0.7555 \\
\hline 1.0 & & 0.817365 & 0.684338 & 0.81738 & 0.68434 & 0.8174 & 0.6844 \\
\hline 1.5 & & 0.716959 & 0.601289 & 0.71694 & 0.60129 & 0.7171 & 0.6015 \\
\hline 2.0 & & 0.647145 & 0.543133 & 0.64713 & 0.54310 & 0.6474 & 0.5435 \\
\hline 0.2 & 0.2 & 1.15589 & 0.956065 & 1.15591 & 0.95607 & 1.1559 & 0.9558 \\
\hline & 0.5 & 1.1441 & 0.96893 & 1.14411 & 0.96893 & 1.1439 & 0.9689 \\
\hline & 0.7 & 1.13961 & 0.973789 & 1.13961 & 0.97378 & 1.1394 & 0.9734 \\
\hline & 1.0 & 1.13481 & 0.978926 & 1.13482 & 0.97892 & 1.1353 & 0.9783 \\
\hline
\end{tabular}

TABLE 2: Comparison of $-\theta^{\prime}(0)$ for various values of $k_{1}$ and $\operatorname{Pr}$ when $m=1.0$ and $\lambda_{T}=0.2$.

\begin{tabular}{|c|c|c|c|c|c|c|c|}
\hline \multirow{3}{*}{$k_{1}$} & \multirow{3}{*}{$\operatorname{Pr}$} & \multicolumn{6}{|c|}{$-\theta^{\prime}(0)$} \\
\hline & & \multicolumn{2}{|c|}{ Present results } & \multicolumn{2}{|c|}{ Li et al. [2] } & \multicolumn{2}{|c|}{ Hayat et al. [43] } \\
\hline & & Assisting flow & Opposing flow & Assisting flow & Opposing flow & Assisting flow & Opposing flow \\
\hline 0.0 & 0.2 & 0.441936 & 0.423511 & 0.44198 & 0.42351 & 0.4420 & 0.4235 \\
\hline 0.2 & & 0.427847 & 0.409582 & 0.42606 & 0.40958 & 0.4261 & 0.4094 \\
\hline 0.5 & & 0.409971 & 0.394995 & 0.40990 & 0.39499 & 0.4097 & 0.3939 \\
\hline 0.7 & & 0.401769 & 0.387536 & 0.40177 & 0.38753 & 0.4018 & 0.3875 \\
\hline 1.0 & & 0.391855 & 0.378372 & 0.39189 & 0.37837 & 0.3920 & 0.3785 \\
\hline 1.5 & & 0.37922 & 0.366516 & 0.37922 & 0.36652 & 0.3793 & 0.3667 \\
\hline 2.0 & & 0.372174 & 0.35721 & 0.36944 & 0.35729 & 0.3698 & 0.3578 \\
\hline 0.2 & 0.2 & 0.427847 & 0.409582 & 0.42606 & 0.40958 & 0.4261 & 0.4094 \\
\hline & 0.5 & 0.60841 & 0.587526 & 0.60841 & 0.58753 & 0.6082 & 0.5874 \\
\hline & 0.7 & 0.69073 & 0.668207 & 0.69073 & 0.66820 & 0.6903 & 0.6678 \\
\hline & 1.0 & 0.788621 & 0.764356 & 0.78862 & 0.76435 & 0.7876 & 0.7669 \\
\hline
\end{tabular}

TABLE 3: Comparison of $f^{\prime \prime}(0)$ for various values of $k_{1}$ and $\operatorname{Pr}$ when $m=1.0$ and $\lambda_{T}=1.0$.

\begin{tabular}{cccccc}
\hline \multicolumn{1}{c}{$k_{1}$} & \multicolumn{2}{c}{ Present results } & $f^{\prime \prime}(0)$ & & Li et al. [2] \\
& & Assisting flow & Opposing flow & Assisting flow & Opposing flow \\
\hline 0.0 & 1.0 & 1.67542 & 0.731431 & 1.67544 & 0.73141 \\
& 1.5 & 1.64064 & 0.775354 & 1.64060 & 0.77535 \\
& 5.0 & 1.54289 & 0.894131 & 1.54287 & 0.89413 \\
0.5 & 1.0 & 1.20508 & 0.531869 & 1.20508 & 0.53186 \\
& 1.5 & 1.18764 & 0.559115 & 1.18764 & 0.63414 \\
1.0 & 5.0 & 1.13575 & 0.634145 & 1.13575 & 0.43579 \\
& 1.0 & 1.00314 & 0.435724 & 1.00314 & 0.45675 \\
& 1.5 & 0.990924 & 0.456753 & 0.99092 & 0.51532 \\
\hline
\end{tabular}

For example, in order to find the optimal values of $\hbar$, the residual error for the HAM 20th order of solution is presented in Figure 5.

\section{Results and Discussion}

In this paper the mixed convection of a steady and incompressible two-dimensional viscoelastic fluid flow over a wedge surface is studied. To the best of authors' knowledge, the current paper is the first paper which presents a similarity solution and includes the effects of buoyancy parameter in mixed convective flow over a horizontal wedge surrounded by the viscoelastic fluid. In this paper, the effects of involved parameters are taken into account and the velocity and temperature distributions are discussed and plotted analytically. To gain a vast understanding of the results, 


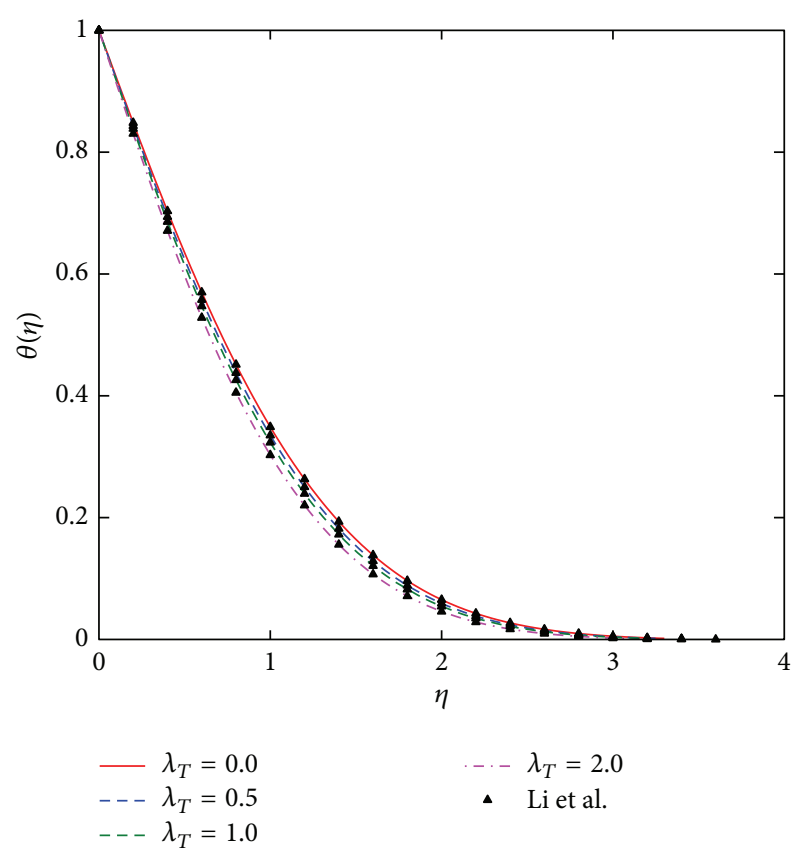

FIGURE 4: Verification of the results $\theta(\eta)$ with the previously published paper by Li et al. when $k_{1}=0.3, m=1.0$, and $\operatorname{Pr}=1.0$.

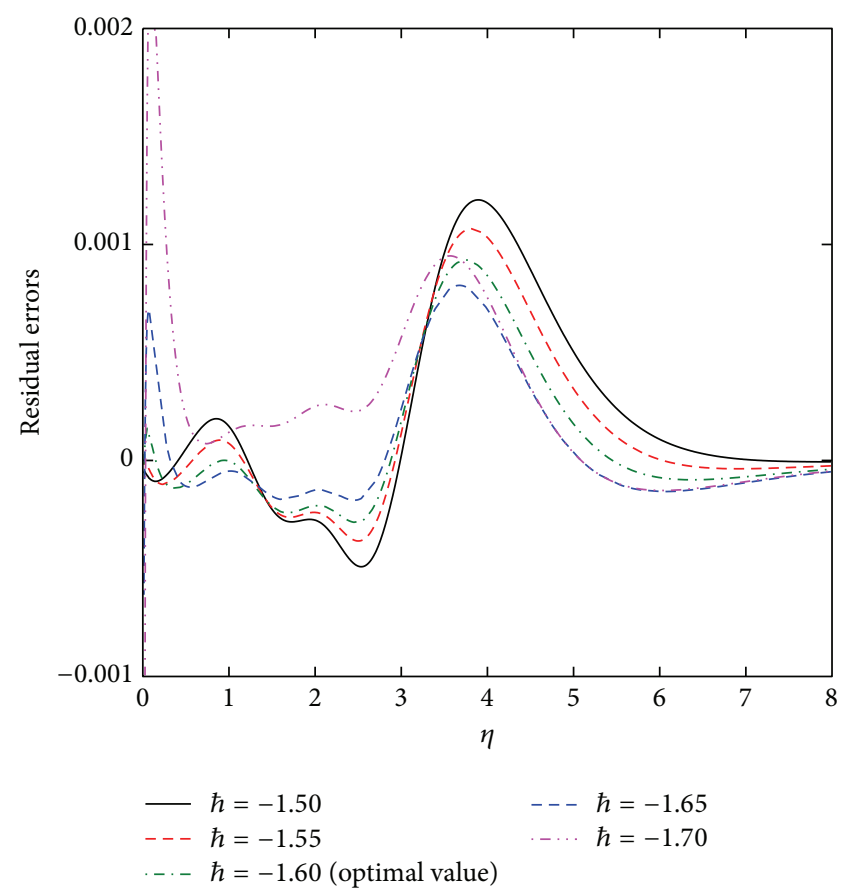

FIGURE 5: The residual errors for temperature profile when $k_{1}=0.8$, $\lambda_{T}=0.5, m=0.5$, and $\operatorname{Pr}=5.0$.

graphical representation is considered for different involved parameters. In Figures 6-13 the effects of different parameters such as viscoelastic parameter, Prandtl number, buoyancy parameter, and the wedge angle parameter are illustrated in detail. Figure 6 denotes the influence of $k_{1}$ on the velocity distribution clearly. Viscoelasticity parameter produces tensile

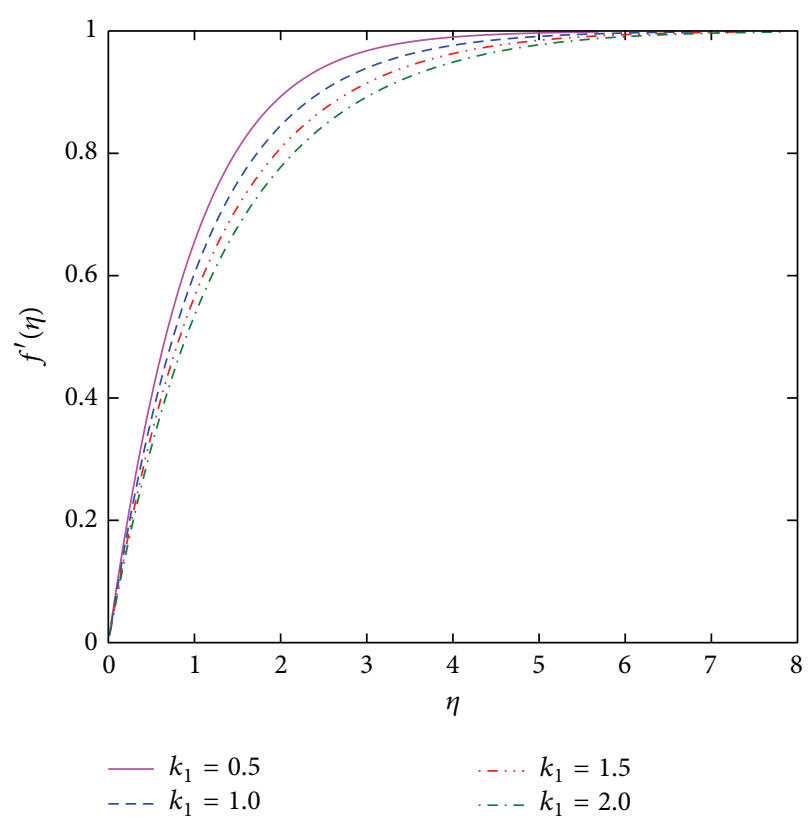

Figure 6: The effect of $k_{1}$ on velocity profile when $\lambda_{T}=0.4, \operatorname{Pr}=1.0$, and $m=0.5$.

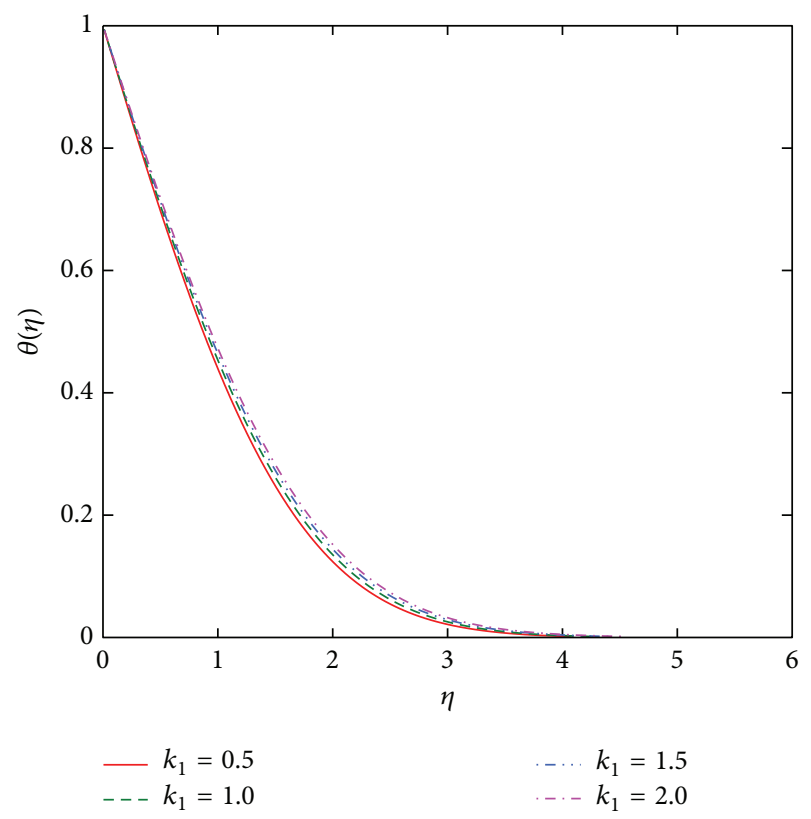

Figure 7: The effect of $k_{1}$ on temperature profile when $\lambda_{T}=0.4$, $\operatorname{Pr}=1.0$, and $m=0.5$.

stress, so the boundary-layer thickness decreases and makes it contract, transversely, and hence velocity decreases. As we anticipate and see in Figure 7, the increase in viscoelastic parameter causes the temperature to increase. The increase in $k_{1}$ leads to increase in wall temperature gradient and the nondimensional temperature distribution is enhanced.

The influence of $\lambda_{T}$ on the velocity and temperature profiles is plotted in Figures 8 and 9. The only term in 
TABle 4: Comparison of $-\theta^{\prime}(0)$ for various values of $k_{1}$ and Pr when $m=1.0$ and $\lambda_{T}=1.0$.

\begin{tabular}{|c|c|c|c|c|c|}
\hline \multirow{3}{*}{$k_{1}$} & \multirow{3}{*}{$\operatorname{Pr}$} & \multicolumn{4}{|c|}{$-\theta^{\prime}(0)$} \\
\hline & & \multicolumn{2}{|c|}{ Present results } & \multicolumn{2}{|c|}{ Li et al. [2] } \\
\hline & & Assisting flow & Opposing flow & Assisting flow & Opposing flow \\
\hline \multirow[t]{3}{*}{0.0} & 1.0 & 0.870746 & 0.731412 & 0.87078 & 0.73141 \\
\hline & 1.5 & 1.00763 & 0.859144 & 1.00763 & 0.85914 \\
\hline & 5.0 & 1.53493 & 1.36374 & 1.53493 & 1.36373 \\
\hline \multirow[t]{3}{*}{0.5} & 1.0 & 0.790331 & 0.673927 & 0.79033 & 0.67393 \\
\hline & 1.5 & 0.913581 & 0.787728 & 0.91358 & 0.78773 \\
\hline & 5.0 & 1.39082 & 1.23669 & 1.39082 & 1.23669 \\
\hline \multirow[t]{3}{*}{1.0} & 1.0 & 0.748488 & 0.640544 & 0.74848 & 0.64054 \\
\hline & 1.5 & 0.864532 & 0.746967 & 0.86453 & 0.74697 \\
\hline & 5.0 & 1.31464 & 1.16674 & 1.31464 & 1.16674 \\
\hline
\end{tabular}

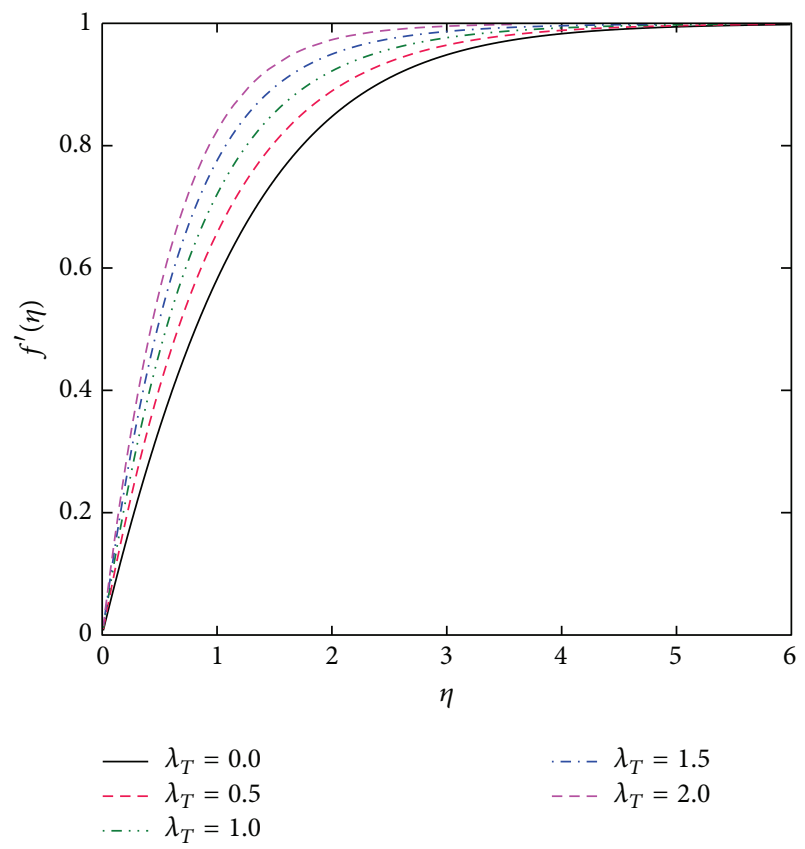

Figure 8: The effect of $\lambda_{T}$ on velocity profile when $k_{1}=0.6, \operatorname{Pr}=1.0$, and $m=0.5$.

momentum equation which causes the energy and momentum equations to be coupled is the buoyancy parameter. When $\lambda_{T}$ increases, the effect of the temperature field on the velocity field will be invigorated and consequently both the velocity and the boundary-layer thickness augment, as shown in Figure 8. This parameter indicates the effect of free convection and buoyancy parameter in equation systems and so reinforces the influence of thermal variations on the velocity component. The buoyancy parameter increases the effect of convection on velocity control. It is worth mentioning that the buoyancy force and favorable pressure gradient have the same impact on velocity in the boundarylayer region and accelerate the fluid. Figure 9 illustrates the effect of $\lambda_{T}$ on temperature distribution. It is clearly depicted that the thermal boundary-layer thickness decreases with the increase in buoyancy parameter because of increase in heat

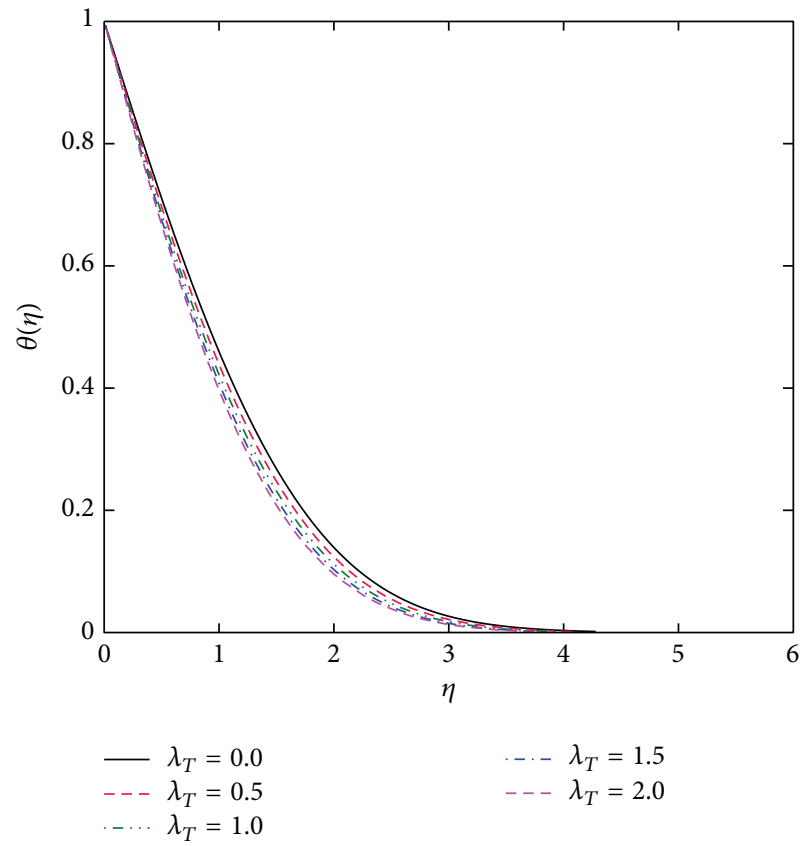

Figure 9: The effect of $\lambda_{T}$ on temperature profile when $k_{1}=0.6$, $\operatorname{Pr}=1.0$, and $m=0.5$.

transfer rate. If we want to illustrate the effect of buoyancy parameter in detail, we should go through this parameter. Based on its definition, the thermal buoyancy parameter is the ratio of buoyancy force to viscous force, so the increase in its value suggests a notable increase in the flow velocity. The Grashof number accelerates the fluid, so the velocity and the boundary-layer thickness increase with the increase in $\lambda_{T}$, as shown in Figure 8 due to this fact that the equations are coupled together only by the buoyancy parameter. In fact the buoyancy force acts like a favorable pressure gradient and accelerates the fluid, so the velocity and the boundary-layer thickness increase with the increase in Grashof number and more production occurs. The buoyancy force leads to the increase in temperature gradient and heat transfer rate and the temperature decreases (Figure 9). When Pr increases, the fluid heat capacity is enhanced and the impact of the thermal 


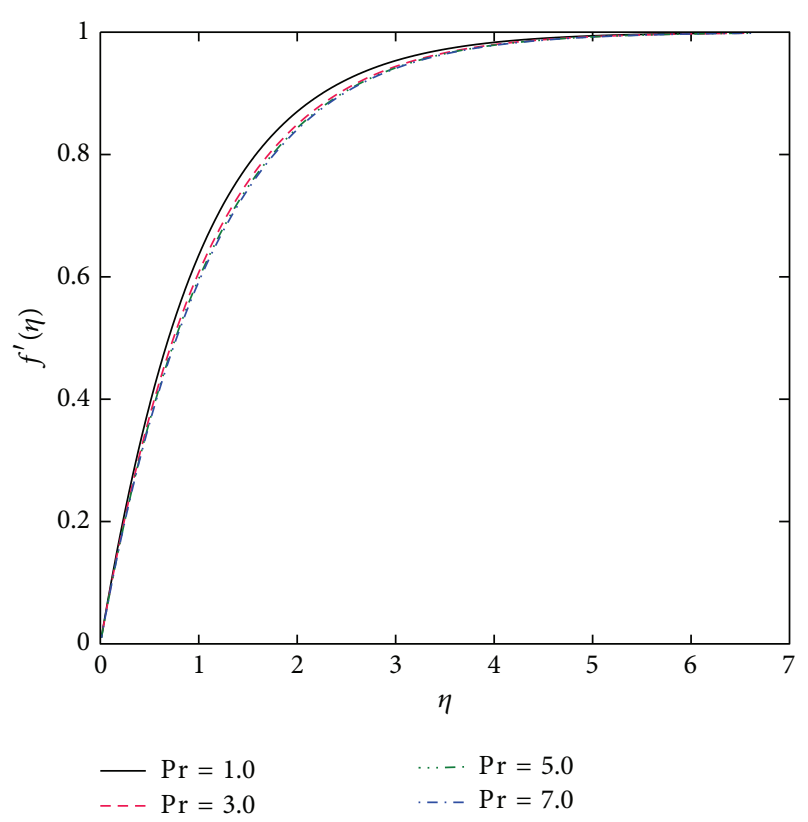

FIgURE 10: The effect of Pr on velocity profile when $k_{1}=0.8, \lambda_{T}=$ 0.5 , and $m=0.5$.

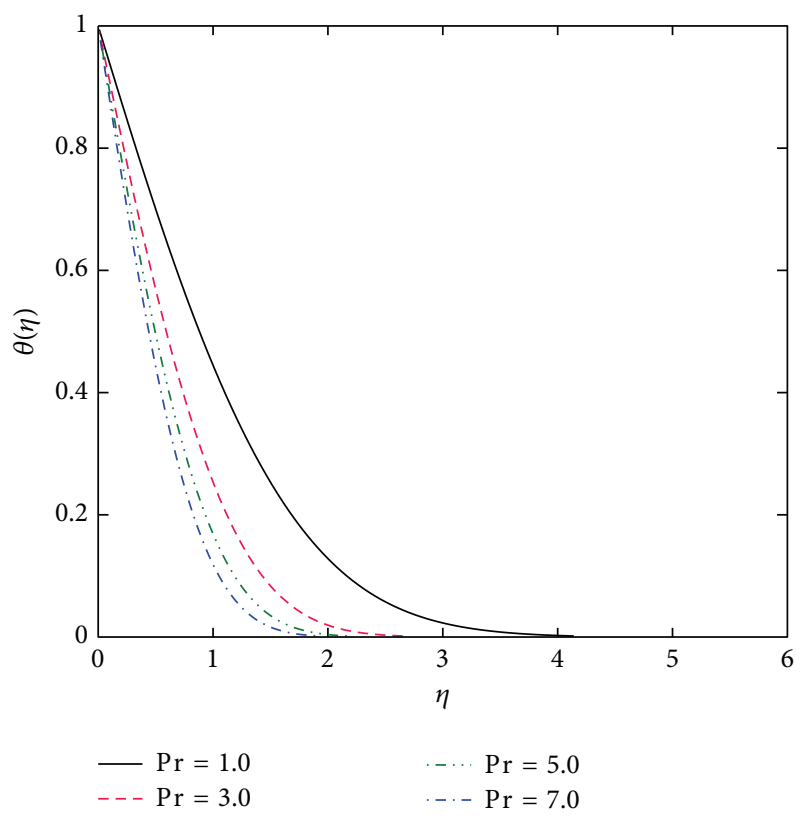

Figure 11: The effect of $\operatorname{Pr}$ on temperature profile when $k_{1}=0.8$, $\lambda_{T}=0.5$, and $m=0.5$.

expansion decreases, so the boundary-layer thickness and velocity distribution decrease (Figure 10). In Figure 11, the effect of Prandtl number on temperature distribution is plotted. Due to the definition of Prandtl number, the ratio of momentum diffusion to thermal diffusion, the thermal diffusion decreases and the fluid thermal capacity increases with the increase in $\mathrm{Pr}$, so the thermal boundary layer becomes thinner and temperature decreases. In Figure 10, the decreasing behavior of velocity component with $\mathrm{Pr}$ is not

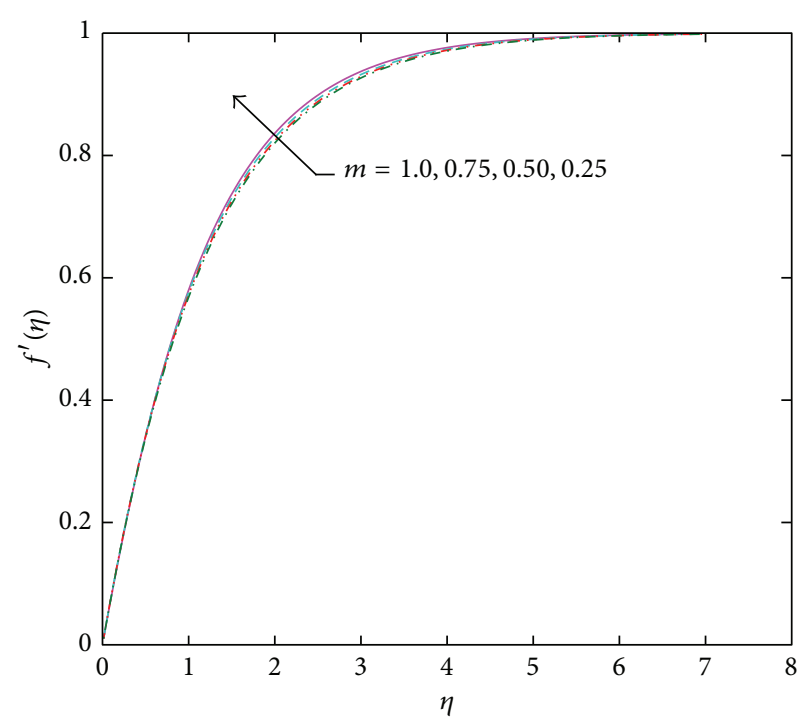

FIGURE 12: The effect of $m$ on velocity profile when $k_{1}=1.0, \lambda_{T}=$ 0.2 , and $\operatorname{Pr}=1.0$.

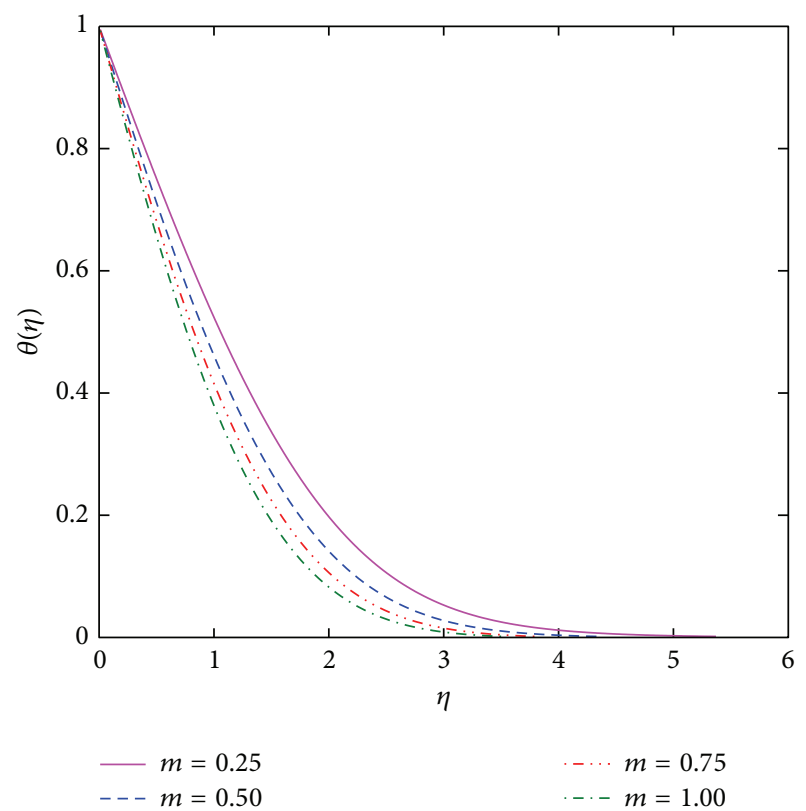

FIGURE 13: The effect of $m$ on temperature profile when $k_{1}=1.0$, $\lambda_{T}=0.2$, and $\operatorname{Pr}=1.0$.

very vivid when Prandtl number varies between 3 and 7 and can be neglected, but the variation in temperature profiles is very distinct and can be easily understood (Figure 11). The effect of parameter $m$ is depicted in Figures 12 and 13. Since the increase in $m$ causes $\beta$ to increase, this parameter represents the influence of wedge angle parameter. As $m$ increases, boundary-layer thicknesses, velocity, and temperature distributions decrease. The variation of velocity distribution with the wedge angle parameter is the same as its behavior with Pr and is not vivid, either. 
In Tables $1-4$ the values of $f^{\prime \prime}(0)$ and $-\theta^{\prime}(0)$ obtained by the 20th order of HAM solution are compared to other different authors' published results. Through these tables, an excellent agreement can be seen between the present results and the previously published papers' results. In Tables 1 and 3 the values of $f^{\prime \prime}(0)$ decrease with the increase in Prandtl number (assisting flow) and the viscoelastic parameter (both assisting and opposing flow regions). Analytical values of wall temperature gradient $-\theta^{\prime}(0)$ for different values of $\operatorname{Pr}$ and $k_{1}$ are computed and presented in Tables 2 and 4 . Analysis of the tabular data shows that the values of viscoelastic parameter reduce the local heat transfer, while the Prandtl number has the reverse effect. The last term of (2) refers to the buoyancy force. The positive case represents buoyancy assisting and negative case is for the buoyancy opposing flow regions. Buoyancy force assists the upper half of the flow field and opposes the lower half of the flow field. Practically in " + " case the external velocity is opposite to the gravity acceleration and in "-" case the external velocity and the acceleration due to gravity have the same direction (for more details, please see [48]).

In this paper, only assisting flow region, the upper half of the flow field, has been taken into account, but in tables, the results of the both cases have been presented for verification purpose.

\section{Conclusion}

In the present paper, a steady incompressible viscoelastic fluid flow over a wedge in the presence of buoyancy force effects has been studied analytically by HAM. This analytical solution shows excellent agreement with the data available in the literature (Tables 1-4 and Figures 3 and 4). As one can easily understand, the values of $f^{\prime \prime}(0)$ decrease with the increase in Prandtl number in assisting flow and vice versa for opposing flow region (Tables 1 and 3 ). These tables clearly show that the skin friction coefficient decreases with the increase in the value of the viscoelastic parameter for both assisting and opposing flows. Analytical values of wall temperature gradient $-\theta^{\prime}(0)$ for different values of Prandtl number $\operatorname{Pr}$ and viscoelastic parameter $k_{1}$ are compared and presented in Tables 2 and 4 . Analysis of the tabular data shows that the viscoelastic parameter reduces the Nusselt number. On the contrary, the effect of Prandtl number is to augment the rate of heat transfer. The dimensionless velocity profiles decrease with the increase in $k_{1}$ and the temperature distribution shows increasing behavior. The effect of increasing the buoyancy parameter is to reduce the thermal boundary-layer thicknesses, but the opposite behavior can be seen for velocity component. The impact of Prandtl number and wedge angle parameter on velocity and temperature profiles is the same. In both cases the decreasing behavior of thermal boundary layer is notable, but the effects of $\operatorname{Pr}$ and $m$ on velocity distribution cannot be distinguished distinctly.

\section{Nomenclature}

$$
\begin{aligned}
& a, b: \text { Constant values } \\
& c_{i}: \quad \text { Arbitrary constant }
\end{aligned}
$$

$c_{P}:$ Specific heat at constant pressure

$g$ : Acceleration due to gravity

$\mathrm{Gr}_{x}$ : Grashof number

$k$ : Thermal conductivity

$k_{1}$ : Viscoelastic parameter

$m$ : Constant value

Pr: Prandtl number

$\mathrm{Re}_{x}$ : Reynolds number

$T: \quad$ Temperature

$u, v$ : Velocity components along and perpendicular to the wedge

$x, y$ : Distance along and perpendicular to the wedge, respectively.

\section{Greek Letters}

$\beta$ : Wedge angle parameter

$\beta_{T}$ : Coefficient of thermal expansion

$\Omega$ : Total angle of the wedge

$\hbar$ : Auxiliary nonzero parameter

$\mathscr{H}$ : Auxiliary function

$\mathscr{L}$ : Auxiliary linear operator

$\mathscr{N}$ : Nonlinear operator

$\eta$ : Similarity variable

$\theta$ : Dimensionless fluid temperature

$\rho:$ Density

$\lambda_{T}$ : Buoyancy parameter

$v$ : Fluid kinematic viscosity

$\psi$ : Stream function.

\section{Subscripts}

e: External condition

$m$ : Mean condition

$w$ : Wall condition

$\infty$ : Infinity condition.

\section{Superscript}

': Differentiation with respect to $\eta$.

\section{Conflict of Interests}

The authors declare that there is no conflict of interests regarding the publication of this paper.

\section{Acknowledgment}

The authors acknowledge the support from the National Research Foundation of South Africa.

\section{References}

[1] X. Su, L. Zheng, X. Zhang, and J. Zhang, "MHD mixed convective heat transfer over a permeable stretching wedge with thermal radiation and ohmic heating," Chemical Engineering Science, vol. 78, pp. 1-8, 2012.

[2] D. Li, F. Labropulu, and I. Pop, "Mixed convection flow of a viscoelastic fluid near the orthogonal stagnation-point on a 
vertical surface," International Journal of Thermal Sciences, vol. 50, no. 9, pp. 1698-1705, 2011.

[3] K. Hiemenz, Die Grenzschicht an einem in den Gleichförmigen Flüssigkeitsstrom Eingetauchten Geraden Kreiszylinder, Weber, Berlin, Germany, 1911.

[4] G. C. Dash and S. C. Behera, "Laminar free convection stagnation heat transfer of a viscoelastic liquid from an isothermal cylinder with an internal source and/or sink," Wear, vol. 81, no. 2, pp. 197-208, 1982.

[5] R. Nazar, N. Amin, D. Filip, and I. Pop, "Stagnation point flow of a micropolar fluid towards a stretching sheet," International Journal of Non-Linear Mechanics, vol. 39, no. 7, pp. 1227-1235, 2004.

[6] M. S. Abel, E. Sanjayanand, and M. M. Nandeppanavar, "Viscoelastic MHD flow and heat transfer over a stretching sheet with viscous and ohmic dissipations," Communications in Nonlinear Science and Numerical Simulation, vol. 13, no. 9, pp. 18081821, 2008.

[7] N. S. Akbar and S. Nadeem, "Analytical and numerical solutions of peristaltic flow of williamson fluid model in an endoscope," Journal of Mechanics in Medicine and Biology, vol. 11, no. 4, pp. 941-957, 2011.

[8] S. Nadeem and N. S. Akbar, "Exact and numerical simulation of peristaltic flow of a non-Newtonian fluid with inclined magnetic field in an endoscope," International Journal for Numerical Methods in Fluids, vol. 66, no. 7, pp. 919-934, 2011.

[9] S. Nadeem and N. S. Akbar, "Numerical solutions of peristaltic flow of Williamson fluid with radially varying MHD in an endoscope," International Journal for Numerical Methods in Fluids, vol. 66, no. 2, pp. 212-220, 2011.

[10] S. Nadeem and N. S. Akbar, "Numerical analysis of peristaltic transport of a tangent hyperbolic fluid in an endoscope," Journal of Aerospace Engineering, vol. 24, no. 3, pp. 309-317, 2011.

[11] P. D. Ariel, "A numerical algorithm for computing the stagnation point flow of a second grade fluid with/without suction," Journal of Computational and Applied Mathematics, vol. 59, no. 1, pp. 9-24, 1995.

[12] T. R. Mahapatra and A. S. Gupta, "Stagnation-point flow of a viscoelastic fluid towards a stretching surface," International Journal of Non-Linear Mechanics, vol. 39, no. 5, pp. 811-820, 2004.

[13] E. Erfani, M. M. Rashidi, and A. B. Parsa, "The modified differential transform method for solving off-centered stagnation flow toward a rotating disc," International Journal of Computational Methods, vol. 7, no. 4, pp. 655-670, 2010.

[14] N. S. Akbar, S. Nadeem, T. Hayat, and A. A. Hendi, "Analytical and numerical analysis of Vogel's model of viscosity on the peristaltic flow of Jeffrey fluid," Journal of Aerospace Engineering, vol. 25, no. 1, pp. 64-70, 2012.

[15] A. Ishak, R. Nazar, N. M. Arifin, and I. Pop, "Mixed convection of the stagnation-point flow towards a stretching vertical permeable sheet," Malaysian Journal of Mathematical Sciences, vol. 1, no. 2, pp. 217-226, 2007.

[16] M. M. Rashidi, D. D. Ganji, and S. M. Sadri, "New analytical solution of stagnation point flow in a porous medium," Journal of Porous Media, vol. 14, no. 12, pp. 1125-1135, 2011.

[17] A. R. M. Kasim, M. A. Admon, and S. Shafie, "Free convection boundary layer flow of a viscoelastic fluid in the presence of heat generation," World Academy of Science, Engineering and Technology, vol. 75, pp. 492-499, 2011.
[18] F. Aman, A. Ishak, and I. Pop, "Mixed convection boundary layer flow near stagnation-point on vertical surface with slip," Applied Mathematics and Mechanics, vol. 32, no. 12, pp. 15991606, 2011.

[19] R. Seshadri, "Unsteady three-dimensional stagnation point flow of a viscoelastic fluid," International Journal of Engineering Science, vol. 35, no. 5, pp. 445-454, 1997.

[20] M. Turkyilmazoglu, "The analytical solution of mixed convection heat transfer and fluid flow of a MHD viscoelastic fluid over a permeable stretching surface," International Journal of Mechanical Sciences, vol. 77, pp. 263-268, 2013.

[21] K. Bhattacharyya and G. C. Layek, "Effects of suction/blowing on steady boundary layer stagnation-point flow and heat transfer towards a shrinking sheet with thermal radiation," International Journal of Heat and Mass Transfer, vol. 54, no. 1-3, pp. 302-307, 2011.

[22] N. Bachok, A. Ishak, and I. Pop, "Melting heat transfer in boundary layer stagnation-point flow towards a stretching/ shrinking sheet," Physics Letters A: General, Atomic and Solid State Physics, vol. 374, no. 40, pp. 4075-4079, 2010.

[23] G. C. Layek, S. Mukhopadhyay, and S. A. Samad, "Heat and mass transfer analysis for boundary layer stagnationpoint flow towards a heated porous stretching sheet with heat absorption/generation and suction/blowing," International Communications in Heat and Mass Transfer, vol. 34, no. 3, pp. 347-356, 2007.

[24] M. Turkyilmazoglu, "Multiple solutions of heat and mass transfer of MHD slip flow for the viscoelastic fluid over a stretching sheet," International Journal of Thermal Sciences, vol. 50, no. 11, pp. 2264-2276, 2011.

[25] M. Turkyilmazoglu, "Multiple solutions of hydromagnetic permeable flow and heat for viscoelastic fluid," Journal of Thermophysics and Heat Transfer, vol. 25, no. 4, pp. 595-605, 2011.

[26] M. Turkyilmazoglu, "Analytic heat and mass transfer of the mixed hydrodynamic/thermal slip MHD viscous flow over a stretching sheet," International Journal of Mechanical Sciences, vol. 53, no. 10, pp. 886-896, 2011.

[27] A. J. Chamkha, M. Mujtaba, A. Quadri, and C. Issa, “Thermal radiation effects on MHD forced convection flow adjacent to a non-isothermal wedge in the presence of a heat source or sink," Heat and Mass Transfer, vol. 39, no. 4, pp. 305-312, 2003.

[28] R. Kandasamy, I. Muhaimin, and A. B. Khamis, "Thermophoresis and variable viscosity effects on MHD mixed convective heat and mass transfer past a porous wedge in the presence of chemical reaction," Heat and Mass Transfer, vol. 45, no. 6, pp. 703-712, 2009.

[29] M. A. Hossain, S. Bhowmick, and R. S. R. Gorla, "Unsteady mixed-convection boundary layer flow along a symmetric wedge with variable surface temperature," International Journal of Engineering Science, vol. 44, no. 10, pp. 607-620, 2006.

[30] S. J. Liao, Beyond Perturbation: Introduction to the Homotopy Analysis Method, vol. 2, Chapman \& Hall/CRC, Boca Raton, Fla, USA, 2004.

[31] S. J. Liao, "On the homotopy analysis method for nonlinear problems," Applied Mathematics and Computation, vol. 147, no. 2, pp. 499-513, 2004.

[32] M. M. Rashidi, M. Ashraf, B. Rostami, M. T. Rastegari, and S. Bashir, "Mixed convection boundary-layer flow of a micro polar fluid towards a heated shrinking sheet by homotopy analysis method," Thermal Science, 2013.

[33] M. Mustafa, T. Hayat, I. Pop, S. Asghar, and S. Obaidat, "Stagnation-point flow of a nanofluid towards a stretching 
sheet," International Journal of Heat and Mass Transfer, vol. 54, no. 25-26, pp. 5588-5594, 2011.

[34] S. Dinarvand, A. Doosthoseini, E. Doosthoseini, and M. M. Rashidi, "Series solutions for unsteady laminar MHD flow near forward stagnation point of an impulsively rotating and translating sphere in presence of buoyancy forces," Nonlinear Analysis: Real World Applications, vol. 11, no. 2, pp. 1159-1169, 2010.

[35] T. Hayat, Z. Abbas, and M. Sajid, "MHD stagnation-point flow of an upper-convected Maxwell fluid over a stretching surface," Chaos, Solitons \& Fractals, vol. 39, no. 2, pp. 840-848, 2009.

[36] S. H. Nourbakhsh, A. A. P. Zanoosi, and A. R. Shateri, "Analytical solution for off-centered stagnation flow towards a rotating disc problem by homotopy analysis method with two auxiliary parameters," Communications in Nonlinear Science and Numerical Simulation, vol. 16, no. 7, pp. 2772-2787, 2011.

[37] M. M. Rashidi and S. Dinarvand, "Purely analytic approximate solutions for steady three-dimensional problem of condensation film on inclined rotating disk by homotopy analysis method," Nonlinear Analysis: Real World Applications, vol. 10, no. 4, pp. 2346-2356, 2009.

[38] M. M. Rashidi, T. Hayat, E. Erfani, S. A. M. Pour, and A. A. Hendi, "Simultaneous effects of partial slip and thermaldiffusion and diffusion-thermo on steady MHD convective flow due to a rotating disk," Communications in Nonlinear Science and Numerical Simulation, vol. 16, no. 11, pp. 4303-4317, 2011.

[39] M. M. Rashidi, S. A. M. Pour, T. Hayat, and S. Obaidat, "Analytic approximate solutions for steady flow over a rotating disk in porous medium with heat transfer by homotopy analysis method," Computers \& Fluids, vol. 54, no. 1, pp. 1-9, 2012.

[40] M. Turkyilmazoglu, "Purely analytic solutions of magnetohydrodynamic swirling boundary layer flow over a porous rotating disk," Computers \& Fluids, vol. 39, no. 5, pp. 793-799, 2010.

[41] M. Turkyilmazoglu, "Analytic approximate solutions of rotating disk boundary layer flow subject to a uniform suction or injection," International Journal of Mechanical Sciences, vol. 52, no. 12, pp. 1735-1744, 2010.

[42] S. Xinhui, Z. Liancun, Z. Xinxin, and S. Xinyi, "Homotopy analysis method for the asymmetric laminar flow and heat transfer of viscous fluid between contracting rotating disks," Applied Mathematical Modelling, vol. 36, no. 4, pp. 1806-1820, 2012.

[43] T. Hayat, Z. Abbas, and I. Pop, "Mixed convection in the stagnation point flow adjacent to a vertical surface in a viscoelastic fluid," International Journal of Heat and Mass Transfer, vol. 51, no. 11-12, pp. 3200-3206, 2008.

[44] T. Hayat, M. Mustafa, and I. Pop, "Heat and mass transfer for Soret and Dufour's effect on mixed convection boundary layer flow over a stretching vertical surface in a porous medium filled with a viscoelastic fluid," Communications in Nonlinear Science and Numerical Simulation, vol. 15, no. 5, pp. 1183-1196, 2010.

[45] S. J. Liao, "An optimal homotopy-analysis approach for strongly nonlinear differential equations," Communications in Nonlinear Science and Numerical Simulation, vol. 15, no. 8, pp. 2003-2016, 2010.

[46] S. J. Liao, Advances in the Homotopy Analysis Method, World Scientific, 2014.

[47] M. M. Rashidi, S. A. M. Pour, and S. Abbasbandy, "Analytic approximate solutions for heat transfer of a micropolar fluid through a porous medium with radiation," Communications in Nonlinear Science and Numerical Simulation, vol. 16, no. 4, pp. 1874-1889, 2011.
[48] A. Ishak, R. Nazar, and I. Pop, "MHD mixed convection boundary layer flow towards a stretching vertical surface with constant wall temperature," International Journal of Heat and Mass Transfer, vol. 53, no. 23-24, pp. 5330-5334, 2010. 


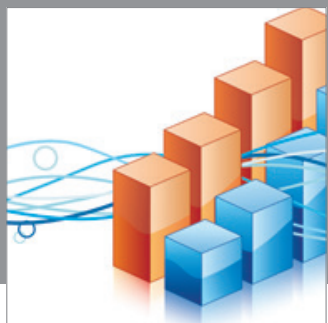

Advances in

Operations Research

mansans

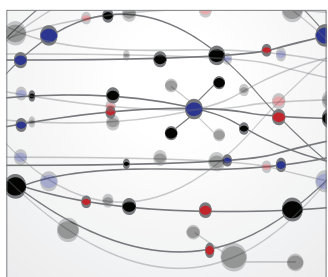

The Scientific World Journal
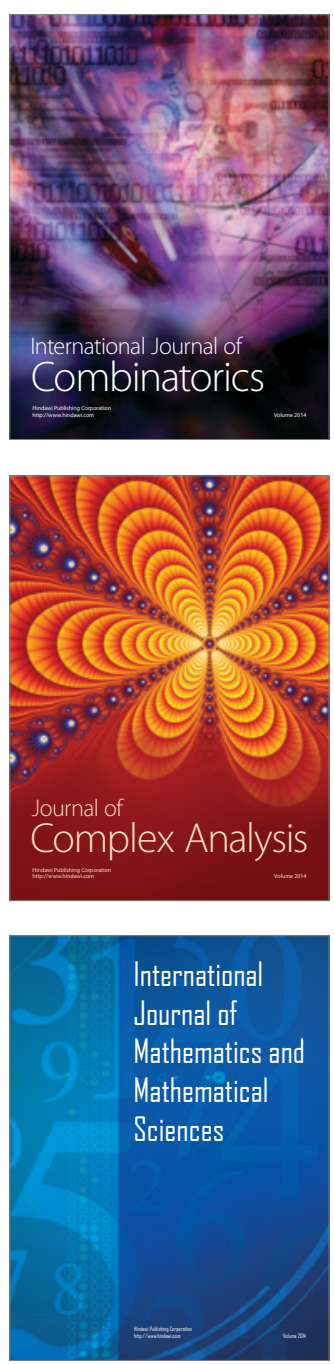
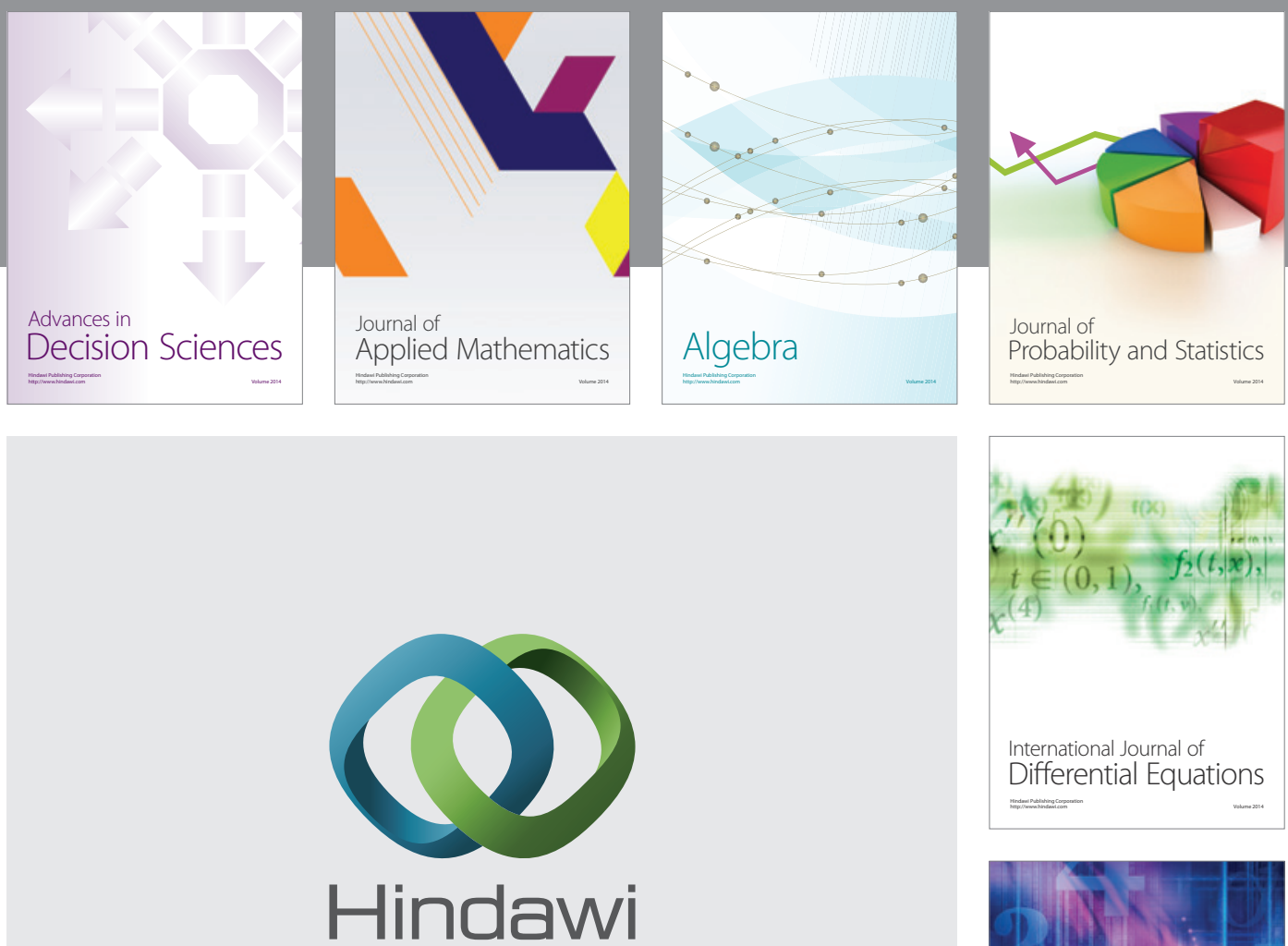

Submit your manuscripts at http://www.hindawi.com
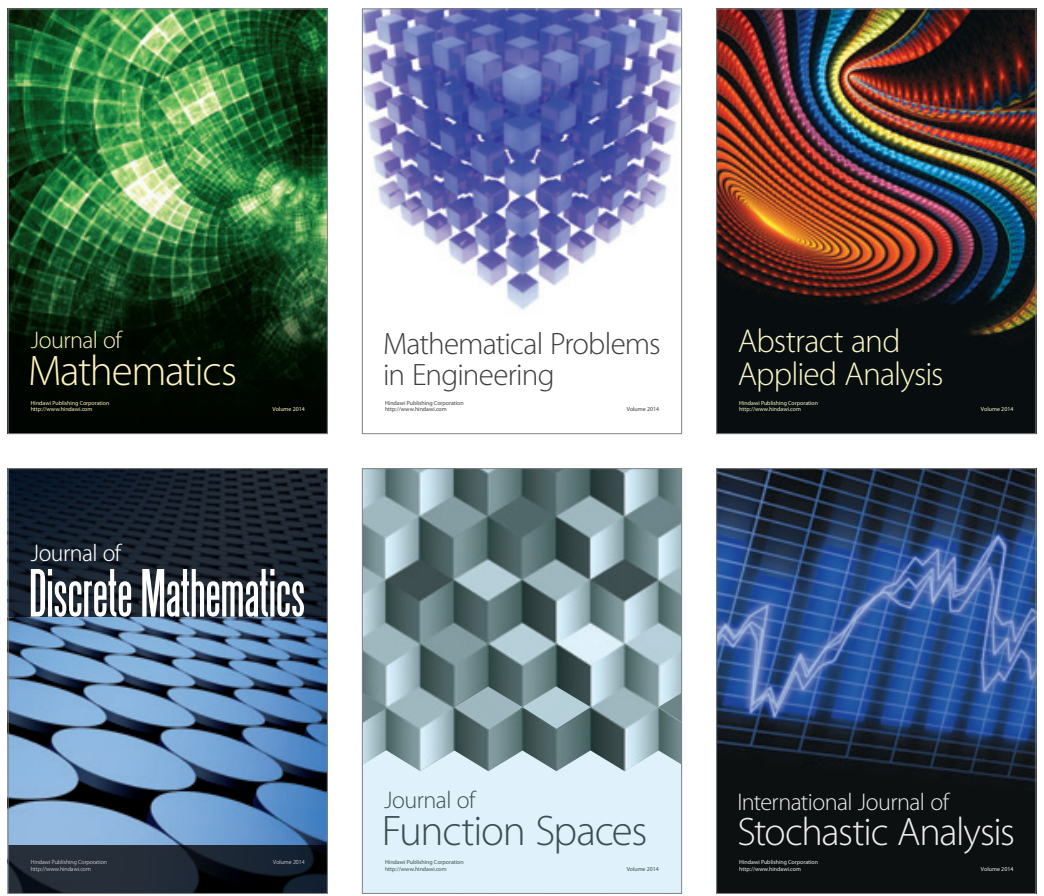

Journal of

Function Spaces

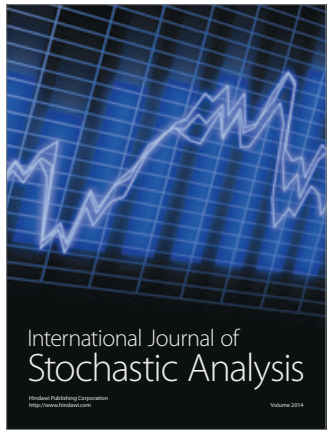

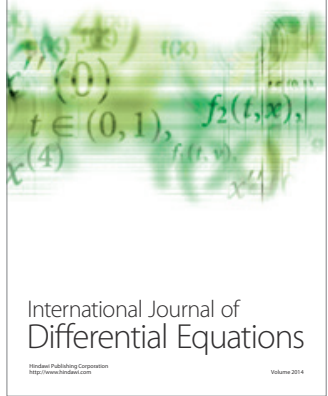
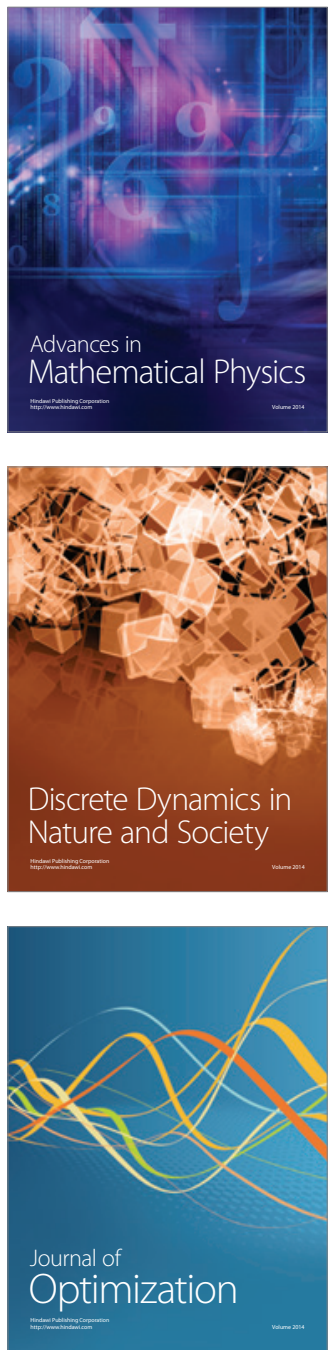